\title{
Research on the Influence of Roadside Billboards on Cognitive Workload of Young Drivers and Traffic Safety
}

\author{
Miljenko MUSTAPIĆ*, Joso VRKLJAN, Vjekoslav JELEČ
}

\begin{abstract}
Based on a vast number of worldwide studies concerning driving behavior and traffic safety, lack of drivers' attention and distraction is recognized as two of the most critical factors for road safety. While roadside advertising is often identified as a potential source of distraction, it has received less attention compared to other types of distractions such as texting or calling while driving. Young drivers, 18 - 25 years old, seem to invest more resources interacting with roadside advertising, suggesting a lower capacity to discriminate between relevant and irrelevant driving information. Combined with lesser driving experience, susceptibility to roadside distractions such as advertising signs, static or changeable, can increase traffic safety risks involving young drivers. Therefore, this study focused on the impact of roadside advertising signs on young drivers, specifically on their cognitive workload while driving in an urban environment cluttered with roadside billboards. The research has been conducted by simultaneously using driving simulator, wireless mobile EEG device for the measurement of the brain's electrical activity, and mobile ETG (eye-tracking glasses) for the measurement of eye movement while driving. The research included 20 young drivers $18-25$ years old. Research results from EEG device showed statistically significant difference in young drivers' cognitive workload related to roadside advertising, with higher cognitive workload while driving in an environment including billboards. Research results from the simulator showed statistically significant driving speed change-drivers accelerating while driving through environment saturated with roadside billboards. Research results from ETG and questionnaire showed three common features of the static roadside billboards that draw more of driver's attention: bigger billboards (megaboards), well-known brands, and provocative design.
\end{abstract}

Keywords: advertising signs; cognitive workload; roadside billboards; traffic safety; young drivers

\section{INTRODUCTION}

Wide range of factors influences driving performance, including fatigue [1], distraction [2], mood [3], driver's experience, personality etc. Among these, distraction is recognized as one of the most critical factors for road safety worldwide [4]. According to scientific research [5], distracted drivers are heavily overrepresented in road traffic crashes. Driver's distractions are usually divided in two groups based on their origin, in-vehicle or external. Any driving non-related tasks that drivers perform in vehicle, such as using mobile phone for texting or calling, interacting with vehicle's infotainment system, calibrating navigation system etc., are considered to be in-vehicle distractions. External distractions consider driver's interaction with the environmental elements outside the vehicle which are not related to driving, such as roadside advertising or landscape.

Beside the division between in-vehicle and external distractions, distraction can also be qualified according to quantified reasons, variables, strength or impact level. Such parameters represent input data that need to be individually detected; causes need to be defined and impacts on drivers need to be determined. When observing distractions as a phenomenon during driving, for the purposes of proper approach, all four basic forms of distraction must be processed [6]:

- visual distraction (looking away from traffic),

- hearing distraction (talking when driving),

- biomechanical distraction (physical activity in a vehicle that is not related to driving),

- cognitive distraction (lack of concentration, mental workload during driving).

Distraction can be and usually is a combination of all four forms. In this paper, cognitive and visual distraction will be processed, with the latter being the most common. While there are no definite scientific conclusions on how much of all driving related information drivers acquire using their vision, there is no doubt that vision and perception of visual input play a crucial part in the driving process [7]. According to Crundall et al. (2006), in most undemanding driving situations drivers often let their eyes wander to irrelevant objects. Percentage estimates of driving time where drivers' attention is directed to scenery and other irrelevant items can vary from $20 \%$ or less, to $50 \%$. These percentages reflect the spare capacity that roadside advertisements are designed to attract, but there is a reasonable concern that advertisements' demand for attention can easily exceed drivers' spare capacity [8].

Scientists have been trying to define distraction through psychology using Neisser or Gestalt theory [9], and the final definition of distraction in the international scientific community was adopted at the first international conference on driving distraction [10] and it reads: Distraction implies diverting attention from driving when a driver temporarily focuses on an object, person, thing or event that is not related to the task of driving, which reduces driver's attention, affects decision-making and consequently leads to risky situations and traffic accidents. Further to this definition, later explanations were given, for example, that distraction does not imply the current state of the driver (fatigue, alcoholism, health conditions, age, etc.), but such conditions favor distraction and that distraction should not result in unwanted outcome but is assumed to significantly increase the risks. Young drivers are especially vulnerable to distraction, because of their driving inexperience, high adoption of communication technology, increased peer involvement, and tendency to take risks [11].

In research on distractions, the only safe way to detect a problem is to measure the consequences of distractions. Consequences can be traffic accidents, driving speed changes, loss of control over the vehicle, and changes of vehicle position within the traffic lane. Such consequences can be measured using various methods, and the most commonly used methods of collecting data used for scientific purposes of research on distraction can be reduced into three main groups: 
- research based on statistics on traffic accidents,

- observation research,

- experimental driving research.

In the context of distracted driving, crash data is acknowledged as the key performance measure for safety [12]. However, there can be one significant limitation in using official traffic accident reports as a data source and that is possible underrepresentation of traffic accidents related to distractions which do not result in heavy injuries and/or fatalities. Underrepresentation of accidents related to distractions can lead to significant understatement of the correlation between distraction and traffic accidents.

Laboratory observations, which include high-fidelity and desktop driving simulators, have been utilized extensively in road safety research, as they are a low risk and low-cost option for studying driving behaviors while controlling different factors [13].

Experimental research using driving simulator combined with EEG and ETG devices to measure cognitive workload and visual distraction caused by advertising, as presented in this study, is the first such research model used up to this date in Croatia.

\section{RESEARCH METHODOLOGY}

Data collection in this research has been conducted combining four different methods:

1. Driving simulator (Fig. 1),

2. EEG device (Fig. 2),

3. ETG device (Fig. 3),

4. Questionnaire.

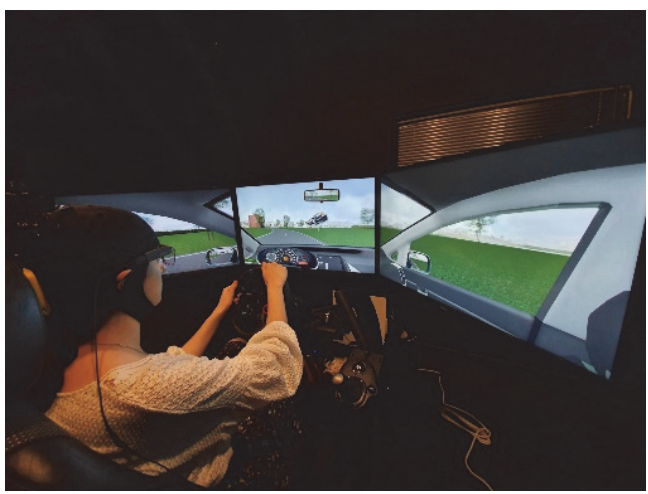

Figure 1 Driving simulator

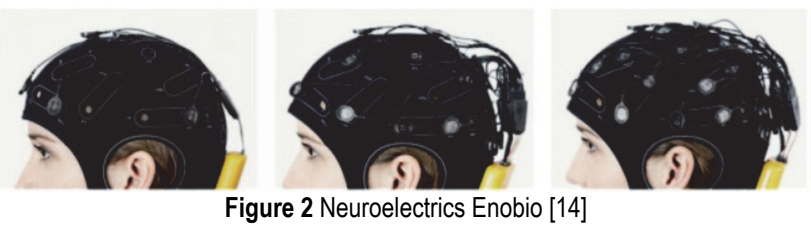

During the first research phase, data were collected while driving a simulator, respondents being additionally equipped with mobile and wireless EEG and ETG devices. The simulator provided data on various driving indicators, such as lateral position (lateral distance between center of front bumper of MainTarget and the centerline of the Rightmost DLANE (DLane(0))), steer error (the deviation between the actual steering wheel angle and the required steering wheel angle), wheel error (the deviation of the front wheel angles from the normative wheel angle, given an ideal path) and speed difference (the difference in $\mathrm{m} / \mathrm{s}$ between the actual velocity and the maximum allowed velocity according to a set of rules used by the robot cars). EEG device provided data on brain's electrical activity and ETG device provided data on number of gazes towards roadside objects, as well as the average duration of each gaze.

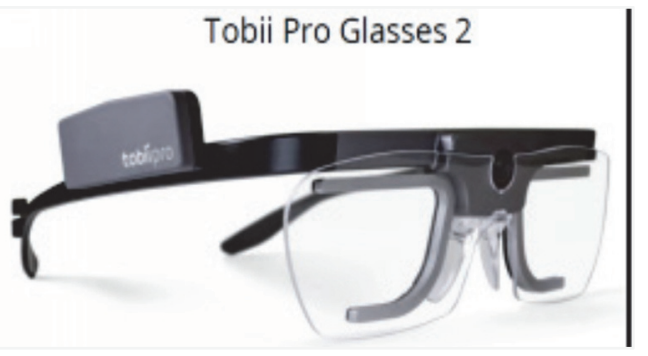

Figure 3 Eye-tracking glasses [15]

During the second research phase, data were collected by applying a questionnaire including demographic variables (gender, age), driving experience variable (how many years of driving, frequency of driving, usual driving environment etc.) and a recall on roadside advertising billboards present on the driving simulator route.

The third phase of the research consisted of data processing, statistical analyses and interpretation.

\subsection{Data Collection}

Simultaneous data collection from the driving simulator, EEG and ETG devices took place during two days in July 2019. For the purpose of the research a driving route in an urban environment was programmed. Driving route was tailor-made for three different phases of driving: 1. Learning phase (baseline, for the study, no billboards), 3 minutes,

2. Driving without roadside billboards present, 3 minutes,

3. Driving with roadside billboards present, 3 minutes.

Under the supervision from the professional in the field of clinical trials of brainwaves and application of eyetracking glasses, every respondent was equipped with mobile wireless EEG and ETG device prior to the simulator drive (Fig. 4)

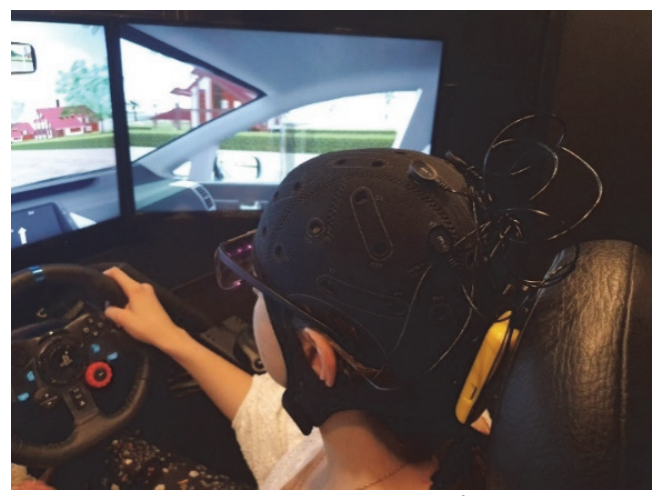

Figure $4 \mathrm{~A}$ respondent driving with the full equipment

In order to exclude the influence of potential fatigue from the data, two groups of respondents were formed. Each group has driven the same route with the difference of having roadside billboards in the second or in the third 
driving phase. Each group consisted of 10 randomly selected respondents.

\section{Group A:}

Condition 1: Learning phase (no billboards, 3 minutes),

Condition 2: Driving with roadside billboards present (3 minutes),

Condition 3: Driving without roadside billboards present (3 minutes).

Group B:

Condition 1: Learning phase (no billboards, 3 minutes),

Condition 2: Driving without roadside billboards present (3 minutes),

Condition 3: Driving with roadside billboards present (3 minutes).

Data collected during learning phase was included in further analyses for the purpose of recognizing and isolating learning effects, which were expected to raise the values of respondents' cognitive workload during their adjustment to the simulator drive.

Immediately after finishing the drive and taking off the equipment, each respondent was asked to fill in a questionnaire. Filling in a questionnaire on a laptop was fairly simple and lasted between 3 and 5 minutes.

\subsection{Data Analysis}

\subsubsection{Sampling}

Non-probability sampling method was used by forming a convenient sample of young drivers, all of them students. Use of sophisticated electronic equipment limited the number of respondents to the ones who do not wear prescription glasses and do not suffer of any chronical disease. Respondents participated in the research voluntarily and did not receive any compensation for their participation.

The sample consisted of 20 young drivers 19 - 24 years old. Average age of the respondents was 22.25. Most of them were between 21 and 23 years old $(60 \%)$.

There were $70 \%$ male and $30 \%$ female respondents.

All of the respondents have a valid driver's license. Most of them $(60 \%)$ got their driver's license 3 or more years ago. There are $35 \%$ of them who have a driver's license for 2 years and 5\% of them who got their driver's license less than a year ago. Half of the respondents (50\%) are in possession of their own vehicle.

Question about the frequency of driving focused on the period of one year prior to the research. Most of the respondents $(65 \%)$ drove every day, $20 \%$ of them once to twice a week and $15 \%$ drove rarely, just once every couple of months.

Most of the respondents frequently drive in an urban environment with the population bigger than 150.000 $(65 \%)$. Further $15 \%$ usually drive in all kinds of environments-urban, rural and motorways. Rest of the respondents drive exclusively in rural or small settlement areas $(10 \%)$ or exclusively on motorways $(10 \%)$.

When it comes to a type of vehicle they drive, specifically the one with manual or automatic gear shift, $55 \%$ of respondents have experience driving with the automatic one (not the usual type of gear shift in Croatia). None of the respondents drove a simulator prior to the research.

\subsubsection{Cognitive Workload-Data Analyses from EEG Device}

$R$ notebook was used for data processing in the following ways:

- Demeaning and detrending in order to exclude artefacts.

- $\quad$ Filtering out low frequencies (the ones below $0.1 \mathrm{~Hz}$ ) by using Butterworth high-pass filter. The default value 0.1 $\mathrm{Hz}$ limit has been set to avoid distortion of the signal [16]. - $\quad$ Filtering out the "noise" by using Butterworth filter in this case is done for the frequency of $50 \mathrm{~Hz}$.

- Excluding the artefact is done by parameter threshold of $400 \mu \mathrm{V}$. All points higher than this threshold are considered extreme values and are excluded from further analysis. To check the quality of signal, percentage of excluded signals has been calculated, because high percentage implicates low quality of signals. Defined threshold value of $400 \mu \mathrm{V}$ is selected in order to maintain typical amplitude of EEG signals for adults, which has a value in a range of 10 to $100 \mu \mathrm{V}$ [17].

- Computation of the Power Spectral Density (PSD) estimate using Fast Fourier Transform (FFT) [18]. Resulting frequencies are in range $\left(0, F_{\mathrm{n}}\right)$, where $F_{\mathrm{n}}(0.5 \times$ sampling Rate) is Nyquist rate of signal processing. The filtered signal is chunked into 2 second time series (with an overlap of 1 second). Each time series is then subdivided in 3 windows of 1 second duration (overlap of 0.5 second) and resulting PSDs are averaged. If less than half of the data points for a given 2 second time series are considered as valid (i.e. not an artefact), the time series is removed from the computation.

- $\quad$ Power band computation by averaging PSD estimate across frequencies of interest (Delta $(1-3 \mathrm{~Hz})$, Theta $(4$ $7 \mathrm{~Hz})$, Alpha (8 - $12 \mathrm{~Hz})$, Beta $(13-25 \mathrm{~Hz})$, Gamma (26 $40 \mathrm{~Hz})$ ). The Neperian Logarithm of these power bands is computed before making graphic review of results.

Theta waves from electrodes F3, Fz, F4 (frontal area), and $\mathrm{P} 3, \mathrm{Pz}, \mathrm{P} 4, \mathrm{Cpl}, \mathrm{Cp} 2$ (parietal area) were observed for cognitive workload since the increase of EEG theta power range reflects the increase in cognitive resources utilization (cognitive workload) [19].

Final data processing for each respondent consisted of the following statistical transformations:

- calculation of average cognitive workload for the whole data set,

- calculation of standard deviation of the cognitive workload for the whole data set,

- calculation of average cognitive workload for each of the three driving phases (learning phase, driving with billboards, driving without billboards),

- standardization of the results (z-values) and the calculation of average $z$-value of cognitive workload for each of three driving phases and for each respondent.

The cognitive workload score was calculated as brain activity in the parietal lobe, according to the data processing method previously mentioned. Due to individual differences in brain activity between respondents while driving and differences in driving experience, the data were transformed into a composite score of the standardized values of each subject, so that the results obtained at different driving phases-learning phase, driving with and without billboards could be compared. 
To compare cognitive workload scores of different driving phases, a pair-sampled $t$-test was conducted in IBM SPSS Statistics software. Following results were obtained: - No statistically significant difference in cognitive workload has been found $(p=0.662)$ between learning phase $(M=-0.007, S D=0.290)$ and driving phase with billboards present $(M=-0.054, S D=0.216)$.

- No statistically significant difference in cognitive workload has been found $(p=0.092)$ between learning phase $(M=-0.007, S D=0.290)$ and driving phase without billboards present $(M=-0.185, S D=0.186)$.

- Statistically significant difference in cognitive workload has been found $(p=0.016)$ between driving phase with billboards present $(M=-0.054, S D=0.216)$ and driving phase without billboards present $(M=-0.185$, $S D=0.186$ )

As expected, learning phase has the highest level of cognitive workload although the difference is not statistically significant. When other two phases were analyzed, it has been found that a driving phase with billboards was connected with higher average workload than a driving phase without billboards.

Since cognitive workload was relatively low in all driving phases, additional analyses of eye-tracking and survey results are done to find out how much attention the respondents paid to billboards. However, the overall cognitive workload while driving can be increased by various unexpected traffic situations which have not been included in simulator drive: waiting during traffic jam, changing the lanes, monitoring traffic rules and adjustment of speed and monitoring the traffic by looking in rearview mirrors. All these could have been the reasons for a relatively low overall results in cognitive workload. Additionally, during a real-life drive much more billboards could be (and in an urban area usually are) present by the roadside with smaller distance between them. In this research respondents had the opportunity to see 37 different billboards 49 times. In real life, driving can also last longer than 3 minutes, which can prolong a period of influence of billboards on drivers, as well as lower drivers' concentration.

Finally, because of the simulator limitations, digital billboards have not been included in the driving route. It can be assumed that these kinds of billboards would represent a bigger distraction, because of the natural reaction to the movement [20].

\subsubsection{Areas of Interest-Data Analyses from Eye-Tracking Glasses}

Eye-tracking is a method of tracking the movement of eyeballs using eye-tracking glasses and software to determine how much interest a content, or a marketing content like billboards, gets from the respondents. Eyetracking glasses measure what a person sees while moving through real space and time-what attracts a gaze the most, and how fast and in what direction is a gaze averted. Areas of Interest analysis is a method of marking, selecting and segmenting areas which attract visual attention. By using this method, it can be determined how many respondents have looked at a specific stimulus and how many fixations there were on a specific stimulus. Fixation is a period of time in which a gaze has been held on an object. Usually, a fixation lasts for 100 - 300 milliseconds. Stimuli that attract the attention more, have larger number of fixations and are noticed by more respondents.

In this research billboards were used as stimuli for eyetracking. In simulator driving route, there were 37 different billboards which could have been seen by the respondents 49 times in total. There were 3 types of (static) billboards:

- standard billboards (24),

- mega-boards (5),

- city lights (20).

In total, respondents fixated their gaze on 14 billboards and 23 billboards did not have a fixation. Mega-boards attracted the most attention. Both the highest number of fixations (11) and the respondents that looked at it, had an advertisement for Ciak Auto (Fig. 5), which has been shown 3 times; one time as a billboard and two times as a mega-board. Subsequent Independent Sample $t$-Test analysis did not result in statistically significant difference in average duration on fixation on this billboard between male and female respondents $(p>0.9)$.

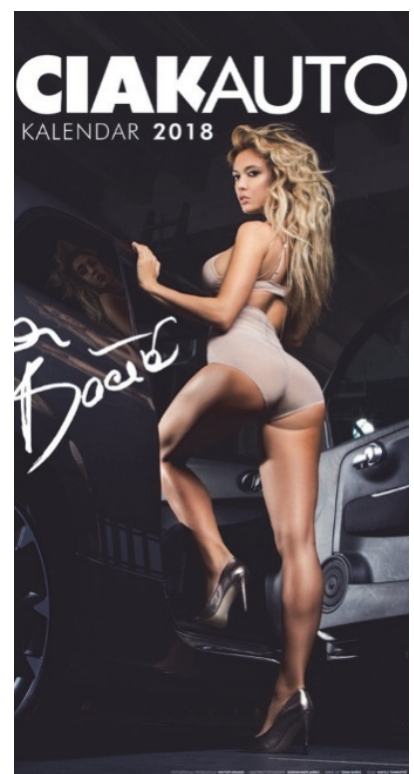

Figure 5 Advertisement which attracted most attention

Advertisements on mega-boards have more fixation on average $(0.65)$ than standard billboards $(0.23)$ and citylights $(0.13)$. There were also more respondents on average who fixated their gaze on mega-boards $(0.73)$ than on standard billboards (0.40) and city-lights (0.27). More detailed results on fixations are shown in Fig. 6 and Fig. 7.

\section{Number of respondents looking at advertisements}

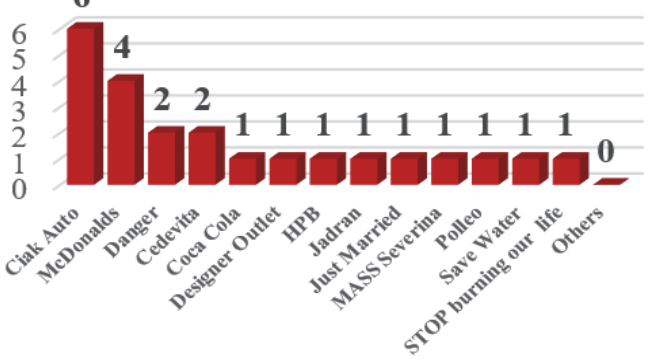

Figure 6 Top-list of advertisements by the number of respondents 


\section{Number of fixations on advertisements}

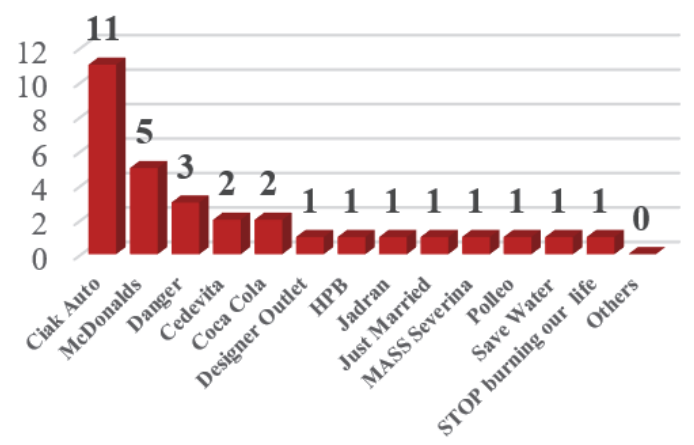

Figure 7 Top-list of advertisements by the number of fixations

It was interesting to see that advertisement "Danger" attracted a fair amount of attention, although such advertisement has never been shown in Croatia. It is an PSA (Public Safety Announcement) ad from a French nonprofit association "Innocence in Danger", which warns the public about the presence of sexual predators who target children through social network apps on smartphones (Fig. $8)$. When we look at the advertisements that got the most attention, it is either a well-known brand, especially to young population, such as McDonald's, Cedevita, Coca Cola, etc., or a design is (sexually) provocative, like the one for Ciak Auto. It can be assumed that the French PSA advertisement attracted more attention than expected because of its provocative design.

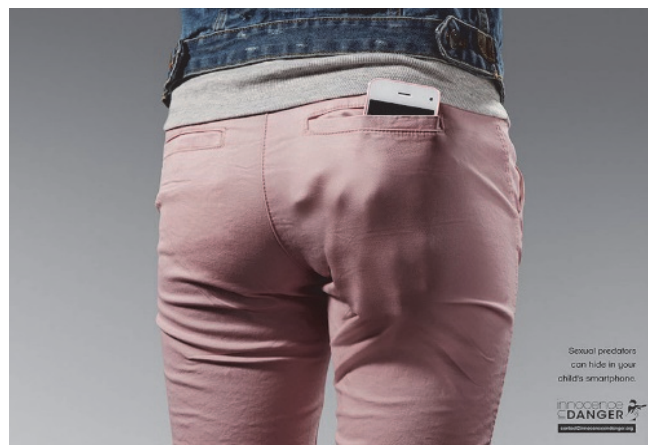

Figure 8 PSA ad from "Innocence In Danger", French non-profit organization

\subsubsection{Data Analyses from Driving Simulator}

For each of 20 respondents we got an Excel document with each row of the workbook representing $1 / 10$ of a second of driving. Path numbers are tagged for each drive as well, to give information on which of the driving phases is taking place during the measurement (learning phase, billboards phase, no billboards phase).

Driving simulator is collecting a broad spectrum of data and four indicators were selected for further analyses:

- Lateral Position,

- Steer Error,

- Wheel Error,

- Speed Difference.

Lateral Position represents lateral distance between center of front bumper of Main Target and the centerline of the Rightmost DLANE (DLane(0)). If the center of the front bumper is to the LEFT of this line: positive values. To the RIGHT of this line: negative values.
Steer Error represents the deviation between the actual steering wheel angle and the required steering wheel angle, in degrees. Required steering wheel angle is computed from vehicle longitudinal velocity, yaw rate and front wheel slip angles.

Wheel Error represents the deviation of the front wheel angles from the normative wheel angle (given an ideal path). The deviation between the actual front wheel angle and the road geometry (normative angle), in degrees ( $0 \ldots$ 360 ). If on a straight road, the normative angle is the angle of the straight segment. If on a curved segment, the normative angle is the tangent of the angle of the line from the center point of the arc to the coordinates of the center of the front bumper.

Speed difference represents the difference (in $\mathrm{m} / \mathrm{s}$ ) between the actual velocity and the maximum allowed velocity according to a set of rules. These rules are the rules used by the robot cars. So, this is the speed difference from the normative model. If this value is positive, the Main Target is driving too fast with respect to the normative model. Value is always $>=0$.

Simulator data were analyzed as follows:

- calculation of total average and standard deviation for each respondent and each indicator (Lateral Position, Steer Error, Wheel Error, Speed Difference),

- calculation of average and standard deviation for each respondent, each indicator and each driving phase (learning phase, billboards, no billboards),

- standardization of the results ( $z$-values) and the calculation of average $z$-value of each indicator for each driving phase.

To compare average $z$-scores on each indicator among all three driving phases, a pair-sampled $t$-test was conducted in IBM SPSS Statistics software.

Following results were obtained:

- Statistically significant difference has been found between learning phase and billboard-phase $(p<0.02)$, and also between learning phase and no-billboards-phase $(p<$ 0.01 ) on Lateral Position, Steer Error and Wheel Error.

- Statistically significant difference has not been found on the same three indicators between billboards-phase and no-billboards-phase $(p>0.05)$.

- Statistically significant difference has been found on Speed Difference between billboard-phase and nobillboard-phase $(p=0.032)$.

Statistically significant difference in average z-scores in Speed Difference between learning phase and billboardsphase, and also between learning phase and no-billboardsphase on indicators that monitor positioning of the vehicle on the road points to expected period of adjusting to simulator driving.

Statistically significant difference in average z-scores between billboards-phase $(z=0.0796)$ and no-billboards phase $(z=-0.0220)$ means that respondents drove faster on average during the billboard-phase $(M=1.695)$ than nobillboard-phase $(M=1.167)$.

\subsubsection{Advertising Recall - Data Analyses from Questionnaire}

Part of the questionnaire that relates to advertising recall should help the interpretation of the results obtained 
by EEG and ETG devices, as well as by driving simulator. Analysis of questionnaire results confirms the ones obtained from ETG device: respondents did not consciously pay much attention to the roadside billboards. When asked how many ads they can spontaneously recall, 4 respondents $(20 \%)$ answered "none". All of the respondents recalled seeing some advertisements, but $20 \%$ of them could not recall which brand, product or service was actually advertised. Other 6 respondents $(30 \%)$ recalled one particular advertisement. Further 5 respondents $(25 \%)$ recalled two particular advertisements. Finally, 3 respondents $(15 \%)$ recalled three advertisements, and two of them (10\%) five and six advertisements respectively.

Four advertisements got the highest spontaneous recall by the number of respondents:

- CIAK Auto (LidijaBačić) - 45\%,

- McDonald's - 35\%,

- Avengers (movie) - 25\%,

- Coca Cola - 15\%.

One thing that these advertisements have in common is that all of them were shown on mega-boards. Furthermore, McDonald's and Coca Cola are well-known brands among young population. CIAK Auto, as we have already shown, probably attracted attention because of sexually provocative design, in addition to being shown on a mega-board. Advertisement for Avengers movie has been shown to respondents once during the drive, on a billboard. It can be assumed that this franchise attracted more attention than other billboards because it also targets young population and it is probably very popular among respondents' peers.

Joint analysis of ETG results and questionnaire results indicates that roadside billboards with sexually provocative designed advertisements attract more attention from young drivers and can represent significant distraction during driving.

Generally speaking, there are few elements that advertisements which attract more attention of young drivers have in common:

- size (bigger billboards attract more attention),

- well-known brands attract more attention,

- sexually provocative design attracts more attention.

\section{CONCLUSION}

Analysis of the research outputs has shown two important things. First of all, it has shown that there is a statistically significant difference in cognitive workload between driving with roadside billboards present and driving without them being present, cognitive workload being bigger with billboards present. Second, the statistically significant difference in deferring from the normative speed between driving with and without roadside billboards present shows that respondents had lesser control on driving speed while driving with billboards present.

These conclusions are worrisome in the context of safe driving, having in mind that Croatian roadsides are heavily "decorated" with avery number, size and type of billboards, both in densely populated areas and around motorways. Even with digital billboards not included in the research, it can be reasonably assumed that they would contribute to the distraction and not reduce it.

In order to lower the risks of driving distraction among Croatian drivers, especially younger and less experienced drivers, more rigorous regulation should be applied to roadside billboards in the sense of their size, type and design.

\section{LIMITATIONS}

Because of the limitations of this research, the results should be indicative for further exploration and should not be applied to the whole population of young drivers. Here are the limitations:

1. Non-probability sampling method was used by forming a convenient sample of young drivers, because of the limited resources. The research should be repeated with a bigger sample, representative of young drivers between age 18 and 26.

2. Also because of the limited resources, simulator driving route was fairly simple and, in that respect, less stressful than driving in real life usually is. No changing lanes was required from the drivers, there were just few traffic signs to monitor, there was no traffic jam etc. Such elements should be added to the simulator driving route in order to make simulator driving experience more similar to the real one.

3. Digital billboards should be included in the simulator driving route because many of those are already on Croatian roadsides. Their presence would add to the similarity with real-life driving experience as well.

4. Finally, limitations of a driving simulator could be overcome by having research participants (respondents) equipped with mobile and wireless EEG and ETG devices, and driving their own vehicle through the designated traffic area.

\section{REFERENCES}

[1] Filtness, A. J., Reyner, L. A., \& Horne, J. A. (2012). Driver Sleepiness-comparison between young and older men during a monotonous afternoon simulated drive. Biological Psychology, 89(3), 580-583. https://doi.org/10.1016/j.biopsycho.2012.01.002

[2] Regan, M. A., Hallett, C., \& Gordon, C. P. (2012). Driver distraction and driver inattention: definition, relationship and taxonomy. Accident Analysis and Prevention, 43(5), 17711781. https://doi.org/10.1016/j.aap.2011.04.008

[3] Rhodes, N., Pivik, K., \& Sutton, M. (2015). Risky driving among young male drivers: the effects of mood and passengers. Transport Research, Part F: Traffic Psychological Behaviour, 28, 65-76. https://doi.org/10.1016/j.trf.2014.11.005

[4] World Health Organisation (WHO) (2011). Mobile phone use: a global problem of driver distraction, World Health Organisation, Geneva, Switzerland.

[5] Beanland, V., Fitzharris, M., Young, K. L., \& Lenne, M. G. (2013). Driver inattention, and driver distraction in serious casualty crashes: data from the Australian National Crash Indepth Study. Accident Analysis and Prevention, 54, 99-107. https://doi.org/10.1016/j.aap.2012.12.043

[6] Ranney, T. A., Garrott, W. R., \& Goodman, M. J. (2000). Driver distraction research: past, present and future. National Highway Traffic Safety Administration.

[7] Topolšek, D., Areh, I., \& Cvahte, T. (2016). Examination of driver detection of roadside traffic signs and advertisements 
using eye tracking. Transp. Res. Part F: Traffic Psychol. Behaviour, 43, 212-224.

https://doi.org/10.1016/j.trf.2016.10.002

[8] Crundall, D., Van Loon, E., \& Underwood, G. (2006). Attraction and distraction of attention with roadside advertisements. Accident Analysis and Prevention, 38 (4), 671-677. https://doi.org/10.1016/j.aap.2005.12.012

[9] Misokefalou, E. (2014). Investigation into and modelling of factors influencing drivers' attention and impacts on road safety. Ph.D. Thesis, University of Thessaly, Volos, Greece.

[10] Regan, M. A., Lee, J. D., \& Young, K. L. (2009). Driver distraction: toward a common definition, presentation. $1^{\text {st }}$ International Conference in Driver Distraction and Inattention, Gothenburg, Sweden.

[11] Buckley, L., Chapman, R., \& Sheenan, G. (2014). Young Driver Distraction: State of the Evidence and Directions for Behavior Change Programs. Journal of Adolescent Health, 54(5), Supplement, s16-s21. https://doi.org/10.1016/j.jadohealth.2013.12.021

[12] Oviedo-Trespalacios, O., Haque, M. M., King, M., \& Washington, S. (2016). Understanding the impacts of mobile phone distraction on driving performance: a systematic review. Transport Research, Part C - Emerging Technology, 72, 360-380. https://doi.org/10.1016/j.trc.2016.10.006

[13] Oviedo-Trespalacios, O., Tuelove, V., Watson, B., \& Hinton, J. A. (2019). The impact of road advertising signs on driver behaviour and implications for road safety: A critical systematic review. Transportation Research Part A, 85-98. https://doi.org/10.1016/.tra.2019.01.012

[14] See https://www.neuroelectrics.com/solutions/enobio/8

[15] See https://www.tobiipro.com/product-listing/tobii-proglasses-2/

[16] Tanner, D., Kara, M. S., \& Luck S. J. (2015). How inappropriate high-pass filters can produce artifactual effects and incorrect conclusions in ERP. Psychophysiology, 52(8), 997-1009. https://doi.org/10.1111/psyp.12437

[17] Aurlien, H. (2004). EEG Background activity described by a large computerized database. Clinical NeurophysiologyOfficial Journal of the International Federation of Clinical Neurophysiology, 115(3), 665-730. https://doi.org/10.1016/j.clinph.2003.10.019

[18] Takahashi, D. (2019). Fast Fourier transform algorithms for parallel computers. Springer, Singapore. https://doi.org/10.1007/978-981-13-9965-7

[19] Puma, S., Matton, N., Paubel, P. V., Raufaste, E., \& ElYagoubi, R. (2018). Using theta and alpha power to assess cognitive workload in multitasking environments. International Journal of Psychophysiology, 123, 11-120. https://doi.org/10.1016/j.jpsycho.2017.10.004

[20] Stavrinos, D., Mosley, P. R., Wittig, S. M., Johnson, H. D., Decker, J. S., Sisiopiku, V. P., \& Welburn, S. C. (2016). Visual behavior differences in drivers across the lifespan: a digital billboard simulator study. Transport research, Part F: Traffic psychol. Behavior, 41, 19-28.

https://doi.org/10.1016/j.trf.2016.06.001
Contact information:

Miljenko MUSTAPIĆ, dipl. ing.

(Corresponding author)

H2 komunikacije d.o.o.,

Dr. Franje Tuđmana $14 \mathrm{~b}$,

10431 Sveta Nedelja, Croatia

E-mail: miljenkom@gmail.com

Joso VRKLJAN, mr. sc.

Likaceste LLC

Smiljanska 41,

53000 Gospić, Croatia

E-mail: joso.vrkljan@likaceste.hr

Vjekoslav JELEČ, PhD, MD

Clinic Hospital Dubrava,

Avenue Gojko Šušak 6,

10000 Zagreb, Croatia

E-mail: vjelec@kbd.hr 